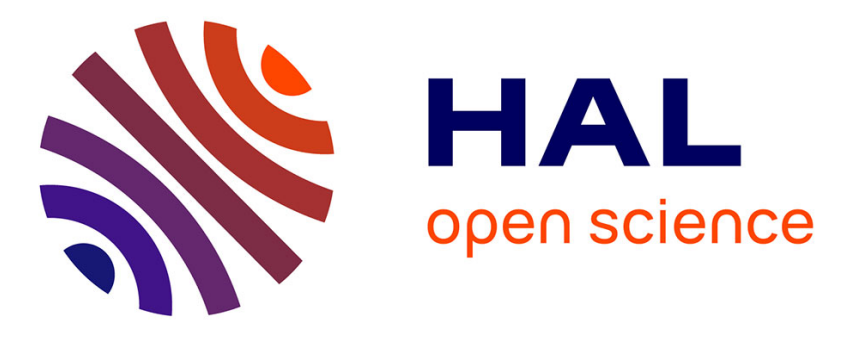

\title{
Oligocellulose from acid hydrolysis: A revisit
}

Feng Jiang, Xin Zhang, Wonseok Hwang, Yoshiharu Nishiyama, Robert Briber, Howard Wang

\section{To cite this version:}

Feng Jiang, Xin Zhang, Wonseok Hwang, Yoshiharu Nishiyama, Robert Briber, et al.. Oligocellulose from acid hydrolysis: A revisit. Applied Surface Science, 2021, 537, pp.147783. 10.1016/j.apsusc.2020.147783 . hal-02951284

\section{HAL Id: hal-02951284 \\ https://hal.science/hal-02951284}

Submitted on 12 Oct 2021

HAL is a multi-disciplinary open access archive for the deposit and dissemination of scientific research documents, whether they are published or not. The documents may come from teaching and research institutions in France or abroad, or from public or private research centers.
L'archive ouverte pluridisciplinaire HAL, est destinée au dépôt et à la diffusion de documents scientifiques de niveau recherche, publiés ou non, émanant des établissements d'enseignement et de recherche français ou étrangers, des laboratoires publics ou privés. 


\section{Oligocellulose from Acid Hydrolysis : A Revisit}

3 Feng Jiang, ${ }^{\mathrm{a}, \mathrm{b}, \#}$, Xin Zhang ${ }^{\mathrm{b}, \#}$, Wonseok Hwang ${ }^{\mathrm{c}}$, Yoshiharu Nishiyama ${ }^{\mathrm{d}}$, Robert M.

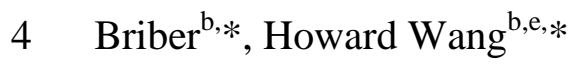

5

$6{ }^{\text {a }}$ Biomass Molecular Engineering Center, Department of Materials Science and

7 Engineering, School of Forestry and Landscape Architecture, Anhui Agricultural

8 University, Hefei, Anhui 230036, China

$9{ }^{\mathrm{b}}$ Department of Materials Science and Engineering, University of Maryland, College Park,

10 Maryland 20742, United States

$11^{\mathrm{c}}$ Department of Chemistry and Biochemistry, University of Maryland, College Park,

12 Maryland 20742, United States

$13{ }^{\mathrm{d}}$ Cermav, CNRS, Grenoble, France

14 e Neutron Science Platform, Songshan Lake Materials Laboratory, Dongguan, Guangdong

$15 \quad 523808$, China

$16{ }^{\#}$ These authors contributed equally to this work 


\section{ABSTRACT}

18 The classical method of producing cellulose oligomers through hydrolysis of

19 microcrystalline cellulose (MCC) in phosphoric acid has been re-examined. Hydrolysis

20 products are fractionated by precipitation to yield two types of cellulose oligomers with

21 different average degrees of polymerization (DP). The number- and weight-averaged

22 molecular weights of the lower DP fraction are $1339 \mathrm{~g} / \mathrm{mol}$ and $1388 \mathrm{~g} / \mathrm{mol}$ respectively

23 corresponding to a polydispersity index of 1.04. The cellulose oligomer forms type-II

24 crystal, and can be dissolved in both alkaline aqueous solutions and dimethyl sulfoxide.

25 The X-ray and neutron small angle scattering of the cellulose oligomers in solution can be

26 described as rigid slabs.

28 Key words: Oligomeric cellulose; acid hydrolysis; polymer physics; small angle neutron

29 scattering 


\section{1. Introduction}

31 Cellulose is the most abundant biomacromolecule on the planet Earth, in which

$32 \beta$-1,4-linked anhydroglucose units (AGU) form long chains that are organized into

33 crystalline nanofibrils [1,2]. Cellulose has found uses in many aspects of everyday life

34 throughout human history, and potential applications in modern technologies [3-7].

35 However, limited by poor processability at the molecular level, the full potential of the

36 renewable biomacromolecules is underutilized. Low molecular mass oligomeric cellulose

37 (OC) can be used as model molecules for a better understanding of the chemical and

38 physical nature of cellulose and its derivatives [8], in addition to finding immediate

39 applications in biomedical and food industries [9, 10]. An easy-to-implement,

40 well-characterized process for cellulose oligomer production is essential for advancing the

41 science and technology of biomacromolecules in general and cellulose in particular.

42 Various methods have been developed to produce OC, including chemical [11] and

43 enzymatic synthesis [12], alkaline [13] and enzymatic degradation [14], acetolysis and

44 deacetylation [15], and acid hydrolysis [16]. For scalable productions, enzymatic

45 depolymerization and acid hydrolysis are considered the more viable methods, with the

46 former potentially useful for the industrial-scale production of water-soluble glucose,

47 cellobiose or cellotetraose as chemical energy sources, and the latter suitable for producing

48 cellulose oligomers with variable degree of polymerization (DP) and polydispersity

49 depending on specific acids and hydrolysis conditions. Hydrolyses in hydrochloric, 
50 sulfuric and nitric acids are often affected by side-reactions to form foreign molecules,

51 resulting in low purity and low yield of cellulose oligomer products [17-19], while

52 phosphoric acid $\left(\mathrm{H}_{3} \mathrm{PO}_{4}\right)$ hydrolyzes cellulose effectively and has lower volatility and

53 toxicity compared to other strong acids. Upon immersing in concentrated $\mathrm{H}_{3} \mathrm{PO}_{4}$,

54 crystalline cellulose fibers dissolve to form a homogeneous solution; subsequently,

55 cellulose chains undergo a three-step hydrolysis process: (1) protonation of the glycosidic

56 oxygen, (2) formation of pyranosyl cations, and (3) addition/heterolytic cleavage of water

57 molecules [8].

58 In a seminal study, Isogai and coworkers have demonstrated $\mathrm{H}_{3} \mathrm{PO}_{4}$-hydrolysis

59 production of two OC fractions with DP peaked at 7 and 15, denoted as DP7 and DP15,

60 respectively [20]. In that original work, the molecular weight distributions were obtained

61 indirectly through intrinsic viscosity and high-performance size-exclusion

62 chromatography (HPSEC) measurements on cellulose tricarbanilate derivatives. In this

63 study, we have revisited the $\mathrm{H}_{3} \mathrm{PO}_{4}$-hydrolysis method and characterized the molecular

64 characteristics of neat OC products using a collection of modern instruments, including

65 matrix-assisted laser desorption/ionization time-of-flight mass spectrometer

66 (MALDI-TOF MS), Fourier-transform infrared (FTIR) spectroscopy, nuclear magnetic

67 resonance (NMR) spectroscopy, wide angle X-ray scattering or diffraction (WAXS, XRD),

68 scanning electron microscopy coupled with energy dispersive X-ray (SEM-EDX) analysis,

69 small angle X-ray and neutron scattering (SAXS and SANS). Due to the higher molecular 
70 weight, DP15 shows poor solubility in common solvents. The preparation of DP7 is fully

71 repeatable, and DP7 can be well dissolved in DMSO, enabling the preparation of cellulose

72 crystals and cellulose derivatives. Therefore, we are mainly focused on DP7 in this work.

73 We obtained a quantitative depiction of the DP7 and confirmed the previous findings, and

74 also revealed the existence of cellulose phosphate in the cellulose oligomer products.

\section{2. Experimental section}

\section{2.1. Materials}

78 Microcrystalline cellulose (MCC, Avicel PH-101), phosphoric acid (85 \% by mass

79 aqueous solution), sodium hydroxide $(\mathrm{NaOH})$, lithium hydroxide ( $\mathrm{LiOH})$, hydrochloric

80 acid $(\mathrm{HCl})$ and acetone were purchased from Sigma-Aldrich, deuterated dimethyl

81 sulfoxide (dDMSO) and deuterium oxide were obtained from Cambridge Isotopes. They

82 were used as received. Following the reported method [20] with slight modifications, 24.0

$83 \mathrm{~g} \mathrm{MCC}$ was mixed with $18 \mathrm{~mL}$ deionized water in a $500 \mathrm{~mL}$ glass bottle, and then $85 \%$

$84 \mathrm{H}_{3} \mathrm{PO}_{4}(450 \mathrm{~mL})$ was introduced. A clear solution formed after about 8 days at room

85 temperature without stirring. After 9 weeks, the dark brown solution was precipitated into

$865 \mathrm{~L}$ deionized water and filtered after 2 hours. The precipitates were collected and denoted

87 as DP15. The filtrate was added into $6 \mathrm{~L}$ methanol and stored overnight. A white

88 precipitate was collected by centrifugation and washed thoroughly with deionized water

89 and acetone, and denoted as DP7. Upon drying the solids under vacuum at room 
90 temperature, the overall yield of cellulose oligomer products from the two precipitates was

91 found at ca. $50 \%$ by mass of the starting MCC, in which the range of yield for DP7 is

$92 \quad 12-20 \%$, and for DP15 at 25-50\%, respectively.

\section{2.2. Characterization}

94 FTIR spectra of pristine MCC, regenerated cellulose from alkaline solution of MCC,

95 and DP7 were recorded on Nicolet Nexus 670 FTIR spectrometer with an attenuated total

96 reflectance (ATR) with a single reflection diamond plate. Regenerated cellulose was

97 prepared by dissolving $\mathrm{MCC}$ in $12 \mathrm{wt} \% \mathrm{NaOH}$ aqueous solution at $-15^{\circ} \mathrm{C}$, precipitating in

980.4 wt $\% \mathrm{HCl}$, followed by continuous water rinse to a neutral $\mathrm{pH}$. NMR measurements

99 were carried out using a Bruker Avance 400 NMR spectrometer on 1 wt $\%$ solution in

100 dDMSO. MALDI-TOF MS spectra were obtained with a Bruker Autoflex Speed

101 spectrometer using 2,5-dihydroxybenzoic acid (DHB) as the matrix in both positive- and

102 negative-ion modes. EDX spectra were collected on a Hitachi SU-70 Field Emission SEM

103 using a Bruker EDX detector. WAXS and SAXS data were recorded using a Xenocs Xeuss

104 instrument with a micro-focus source of the copper $\mathrm{K} \alpha$ radiation $(\lambda=1.5418 \AA)$. SAXS

105 measurements were done on 1-5 wt \% DP7 dissolved in 4 wt \% LiOH aqueous solutions

106 loaded in a $1 \mathrm{~mm}$ diameter capillary with $10 \mu \mathrm{m}$ wall thickness. SAXS data were reduced

107 by subtracting the data from solvent in capillary as background after normalization with the

108 transmission and exposure time. SANS on $1 \mathrm{wt} \% \mathrm{DP} 7 \mathrm{in}$ dDMSO were carried out on the

109 NGB-30 m SANS at the NIST Center for Neutron Research (NCNR), National Institute of 
110 Standards and Technology (NIST). The neutron wavelength $\lambda=6 \AA$ with a spread $\Delta \lambda / \lambda$

111 of $12 \%$. The scattering was measured over a $Q$-range from $\sim 0.003 \AA^{-1}$ to $\sim 0.5 \AA^{-1}$, where

$112 Q$ is the momentum transfer, $Q=\left(\frac{4 \pi}{\lambda}\right) \sin \left(\frac{\theta}{2}\right)$, with $\theta$ being the scattering angle. Data

113 reduction were carried out using the IGOR Pro macros by subtracting blocked beam

114 background, correcting detector efficiency, converting to absolute scattering cross-section,

115 and averaging 2D data into $1 \mathrm{D}$ spectra, $I(Q)$.

\section{3. Results and discussion}

117 3.1. Chemical and crystal structures of $O C$

118 FTIR spectra of MCC, RC and DP7 are shown in Fig. 1. Well-established spectral

119 characteristics can be identified from the MCC spectrum, in which bands in the range from

1203700 to $2995 \mathrm{~cm}^{-1}$ are attributed to hydrogen-bonded $\mathrm{OH}$ stretching. The bands at 2896

$121 \mathrm{~cm}^{-1}, 1641 \mathrm{~cm}^{-1}$, and $1428 \mathrm{~cm}^{-1}$ are assigned to $\mathrm{CH}$ stretching vibrations, $\mathrm{OH}$ bending

122 vibrations of adsorbed water, and $\mathrm{CH}_{2}$ scissoring motion, respectively. The $\mathrm{C}-\mathrm{H}$

123 deformation vibration and C-O stretching vibration can be found at $1367 \mathrm{~cm}^{-1}$ and 1029

$124 \mathrm{~cm}^{-1}$, respectively. The band at $897 \mathrm{~cm}^{-1}$ is attributed to the anomeric $\mathrm{C} 1-\mathrm{H}$ ring valence

125 vibration [21]. The DP7 shows a broader hydrogen-bonded OH stretching band around

$1263336 \mathrm{~cm}^{-1}$ and stronger $897 \mathrm{~cm}^{-1}$ peak, due to the rearrangement of hydrogen bonding and

127 reorientation of hydroxymethyl group in oligocellulose. The infrared spectra of DP7 is

128 almost identical to that of RC and is typical of cellulose II allomorph [22], consistent with

129 literature [23-26]. 


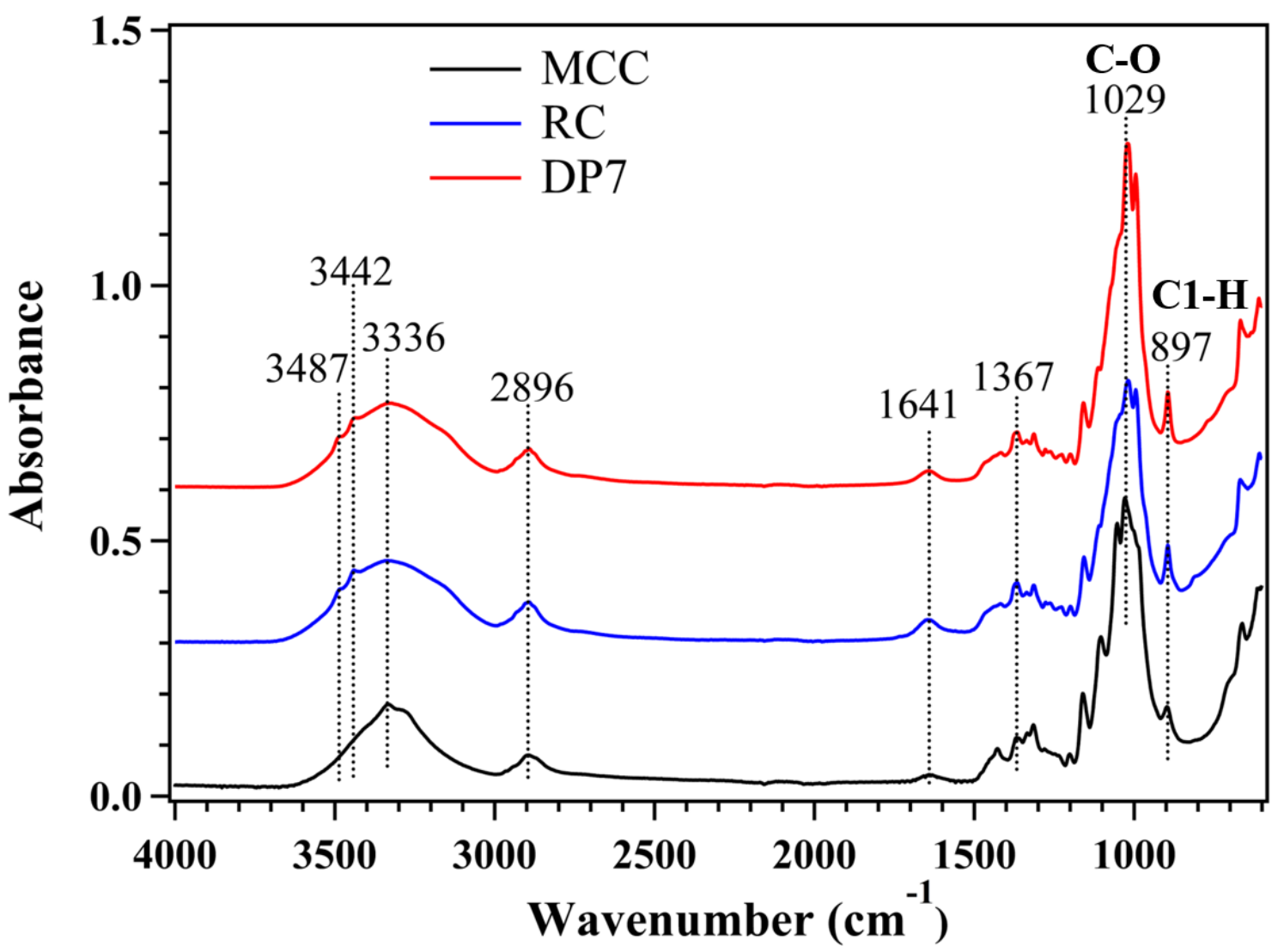

132 Fig. 1. FTIR spectra of microcrystalline cellulose (MCC), regenerated cellulose (RC), and

133 oligocellulose DP7 in the regime of $4000-600 \mathrm{~cm}^{-1}$. The absorbance was normalized to $\mathrm{CH}$

134 stretching at $2896 \mathrm{~cm}^{-1}$. The spectra of RC and DP7 are shifted upward for clarity.

136 Thoroughly washed and dried DP7 powders as shown in Fig. S1 in the Supplementary

137 Information have been measured using X-ray diffraction. As shown in Fig. 2, WAXD

138 pattern of DP7 shows strong diffraction peaks at $2 \theta$ angles of $12.2^{\circ}, 20.0^{\circ}$ and $22.1^{\circ}$, which,

139 according to calculated XRD spectrum using Mercury software from the Cambridge 
140 Crystallographic Data Center (CCDC) based on the cellulose II structure shown in the inset

141 of Fig. 2, correspond to (110), (110) and (020) crystallographic planes [27, 28].

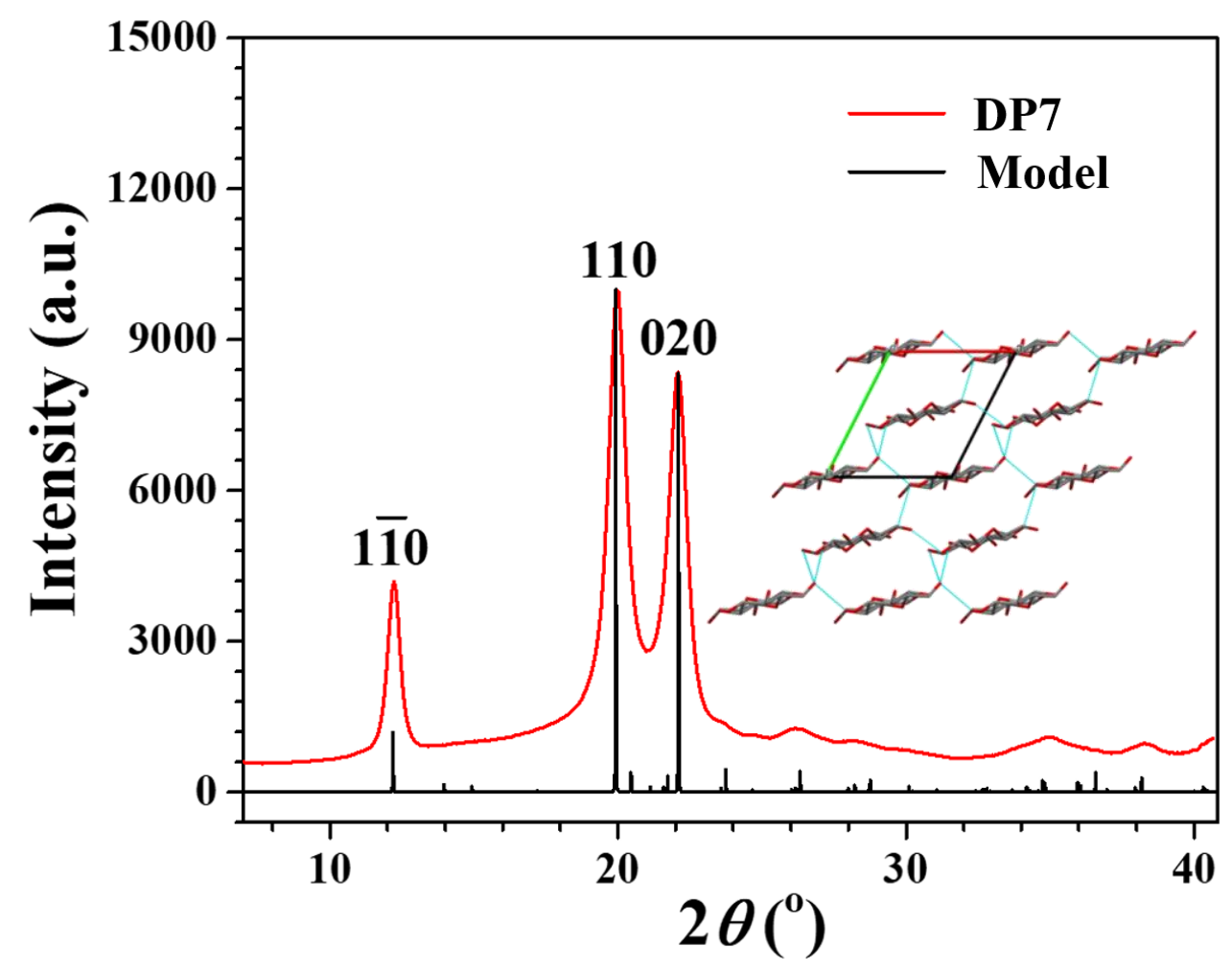

142

143 Fig. 2. The X-ray diffraction pattern of DP7 show prominent diffraction peaks of (110),

144 (110) and (020) crystallographic planes. Computed X-ray diffraction patterns of type-II

145 cellulose crystal structure convoluted with $0.01^{\circ} \mathrm{FWHM}$ is also shown. Inset is the

146 illustration of cellulose II crystal structure viewing from the $\mathrm{c}$ direction.

148 The ${ }^{1} \mathrm{H}$ and ${ }^{13} \mathrm{C}$ NMR spectra of DP7 in dDMSO are shown in Fig. 3 and Fig. S2,

149 respectively. The chemical shifts are identified and annotated on the chemical structure of

150 cellulose on the basis of previous studies [29-32]. As displayed in Fig. 3, the peaks in the

151 4.9-2.8 ppm region are ascribed to the $\mathrm{CH}$ and $\mathrm{CH}_{2}$ protons of the $\mathrm{AGU}$. The weak peaks at 
1524.85 and $4.37 \mathrm{ppm}$ are attributed to the $\alpha$ - and $\beta$-anomeric protons at the reducing end of

153 oligocellulose. The peaks in 6.7-4.4 ppm can be ascribed to the protons of hydroxyl groups.

154 Furthermore, the chemical shifts at 6.30 and $6.64 \mathrm{ppm}$ are due to the hydroxyl protons of $\alpha$ -

155 and $\beta$-anomers at reducing ends of cellulose. Given the quantitative measurements of ${ }^{1} \mathrm{H}$

156 resonance intensity from specific locations on chain ends and all AGU's, the number

157 averaged DP can be calculated as follows [33]

$$
D P n=\frac{I_{\alpha}+I_{\beta}+I_{1}}{I_{\alpha}+I_{\beta}}=1+\frac{I_{1} / I_{\alpha}}{1+I_{\beta} / I_{\alpha}}
$$

159 where $I_{\alpha}$ and $I_{\beta}$ are the integrated intensities of each peak in $(\alpha-\mathrm{H} 1, \beta-\mathrm{H} 1)$ and $(\alpha-\mathrm{OH} 1$,

$160 \beta$-OH1) couples, and $I_{1}$ from the $\mathrm{H} 1^{\prime}, 1^{\prime \prime}$. The averaged $I_{\beta} / I_{\alpha}=1.10$ and $I_{1} / I_{\alpha}=13.9$,

161 resulting in DP 7.6, consistent with the previous study [20].

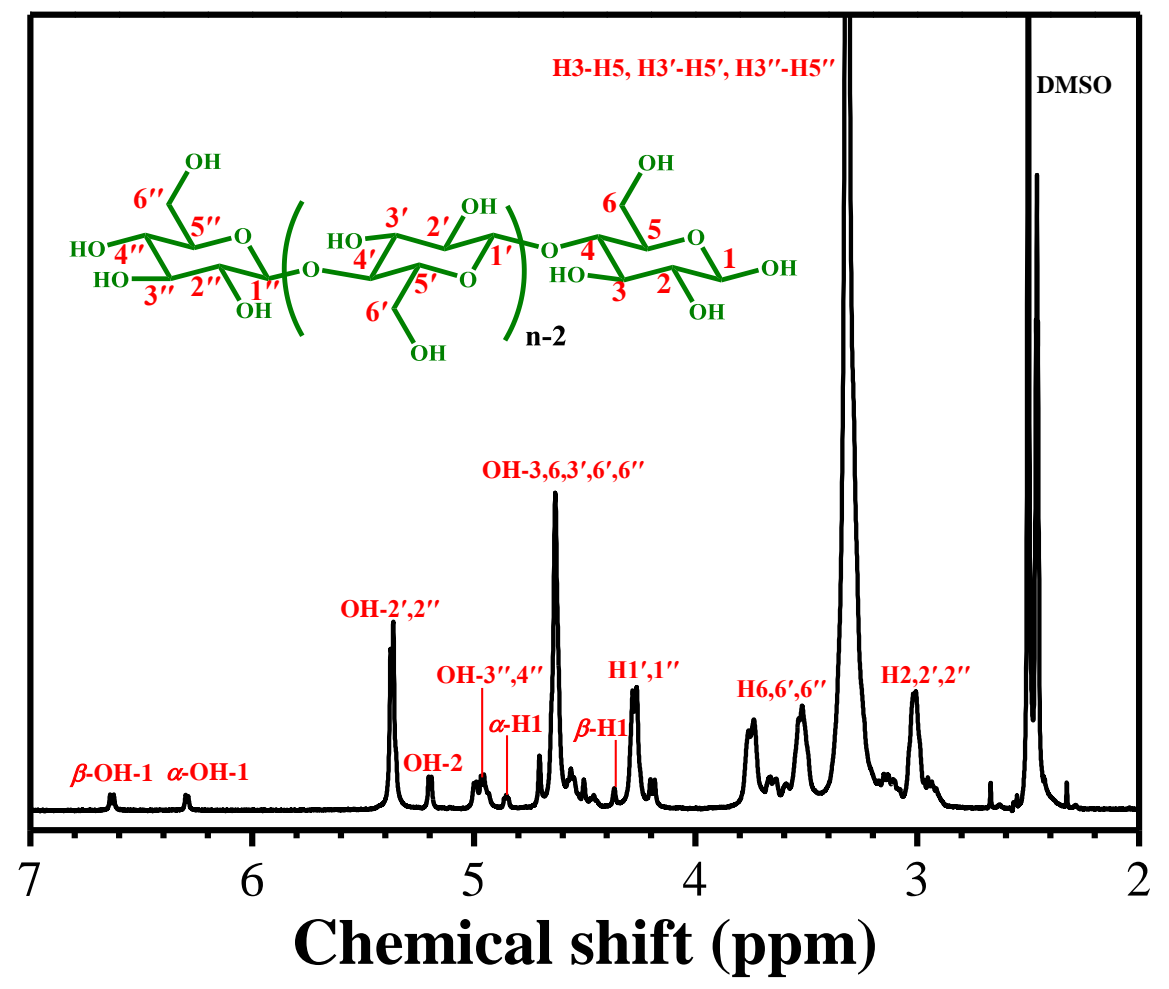


163 Fig. 3. ${ }^{1} \mathrm{H}$ NMR spectrum of DP7 in dDMSO. Characteristic chemical shifts corresponding

164 to specific bonds are annotated on the chemical structure of the cellulose molecule in the

165 inset.

166 3.2. Molecular mass and polydispersity index (PDI) of OC

167 The MALDI-TOF MS spectrum of counts as a function of mass-to-charge $(\mathrm{m} / \mathrm{z})$ ratio in

168 the positive-ion mode is shown in Fig. 4a. Well resolved spectral lines with

169 sub-atomic-unit resolution imply both high purity OC and high-fidelity measurements. The

170 adjacent main lines are separated by $m / z=162$, corresponding to one AGU repeating unit.

171 The primary lines correspond to the sum of the mass of one cellulose molecule containing

$172{ }^{12} \mathrm{C}$ for all carbon atoms and one $\mathrm{Na}$ ion, which is acquired in the process of ionization,

173 while satellite lines separated by one atomic unit to the right of the mainline, as shown in

174 Fig. 4b and c for DP7 and DP11, respectively, corresponding to the inclusion of $1-3{ }^{13} \mathrm{C}$

175 atoms in individual oligocellulose molecules. The distribution profiles of ${ }^{13} \mathrm{C}$ have been

176 examined by comparing the MALDI data with the theoretical prediction according to the

177 random distribution of carbon isotopes, as shown in Fig. 4d, in which the vertical axis is the

178 ratio of the abundance of oligocellulose molecules containing $1-3{ }^{13} \mathrm{C}$ to that of the

179 corresponding neat ${ }^{12} \mathrm{C}$ ones. The diagonal line represents an average ${ }^{13} \mathrm{C}$ abundance of

$1801.084 \pm 0.008 \%$ in the specimen, lower than the overall natural abundance of $1.109 \%$.

181 The percentage histogram of different DPs as color-coded species containing $0-3{ }^{13} \mathrm{C}$ for

182 each DP is shown in Fig. 4e. Data display the dominance of DP7 and DP8 oligocellulose, 
183 and the polydispersity of the specimen spanning over the range of 5-12 AGU. The number-

184 and weight-averaged molecular weight $\left(M_{\mathrm{n}}\right.$ and $\left.M_{\mathrm{w}}\right)$ and PDI value are calculated through

185 a simple summation of all species [34], yielding $M_{\mathrm{n}}=1339 \mathrm{~g} / \mathrm{mol}, M_{\mathrm{w}}=1388 \mathrm{~g} / \mathrm{mol}$, and

186 PDI $=1.04$, respectively. The corresponding number-averaged DP value is 8.15 . The

187 narrow distribution of DP7 is a result of dual precipitations, in water to remove the high DP

188 fraction, and in methanol with water and acetone wash to remove the low DP species and

189 other impurities [16].
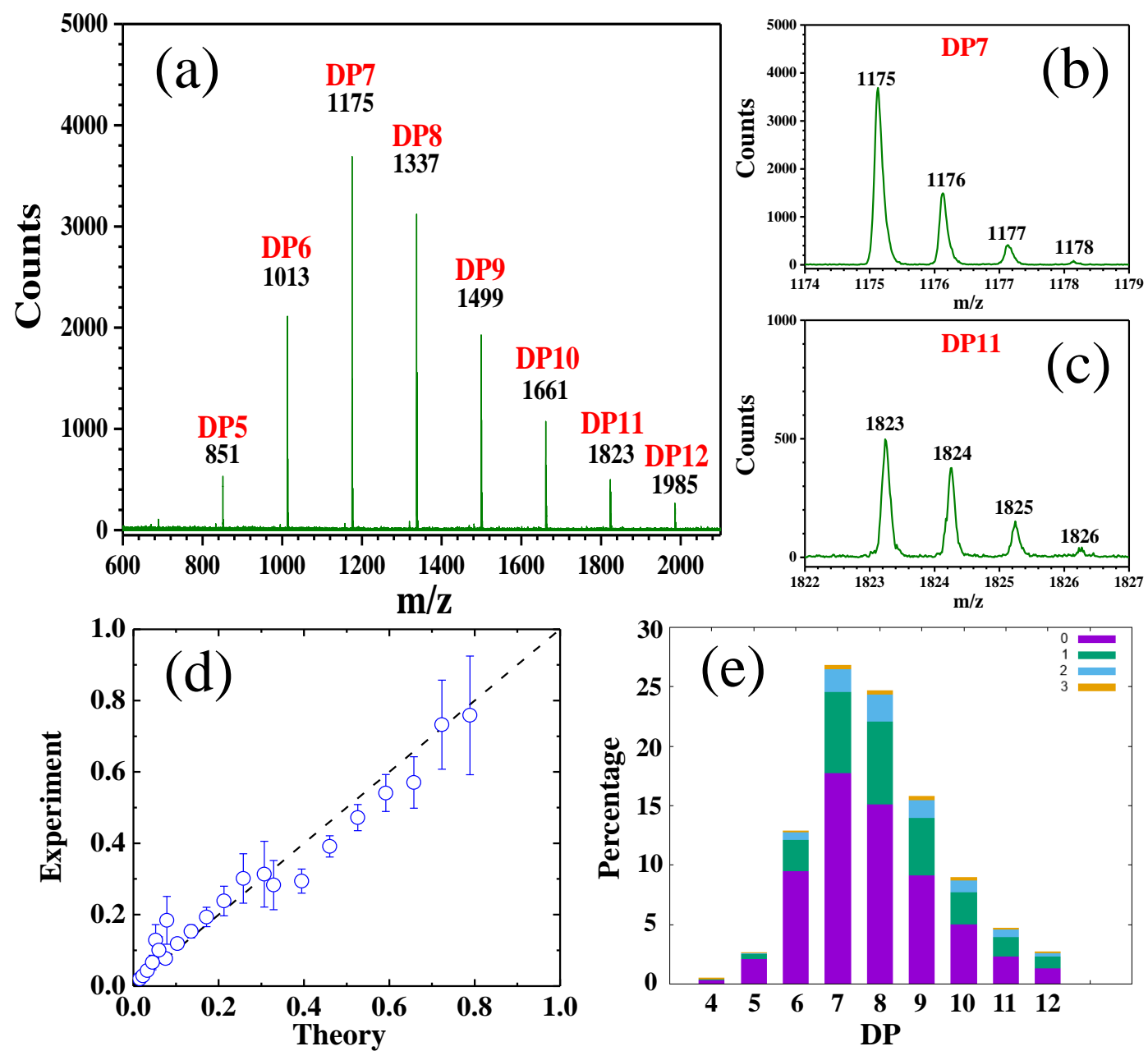

190 
191 Fig. 4. (a) Positive-ion mode MALDI counts as a function of mass-to-charge $(\mathrm{m} / \mathrm{z})$ ratio for

192 the oligocellulose DP7. The molecular mass quantity and corresponding DPs are marked

193 on top of each mainline. The DP has a range of 5-12 and peaks at 7 . The details of spectrum

194 at DP7 (b) and DP11 (c) show satellite peaks with consecutive addition of 1-3 atomic mass

195 number. (d) The ratio of peak areas of ${ }^{13} \mathrm{C}$-containing species to their primary neat- ${ }^{12} \mathrm{C}$

196 peak compared with the prediction based on the random distribution probability. (e)

197 Percentage histogram of different-DP oligocellulose with color-coding for 0 (purple), 1

198 (green), 2 (blue) and 3 (orange) ${ }^{13} \mathrm{C}$ in one cellulose molecule, respectively.

200 MALDI-TOF MS spectrum in negative-ion mode was slightly different, as shown in Fig.

2015 . The lines are separated by the AGU mass of 162, with the mainline quantity equaling to

202 the sum of the mass of one cellulose molecule and one phosphoryl group $\mathrm{PO}_{3}{ }^{-}$(mass value

203 of 79). The DP of cellulose was in the range of 7-15 and peaked at 9, apparently higher than

204 those measured in positive-ion mode. It is conceivable that the measured molecules are

205 cellulose phosphate, which are formed via a C-O-P bond during hydrolysis, and lose one

206 proton and become negatively charged during ionization. Because of the higher solubility

207 of cellulose phosphate in water and protic solvents, it is expected that dual-precipitation

208 and wash process result in an overall higher DP of cellulose phosphate.

209 It is desirable to quantify the amount of phosphorus in cellulose oligomer product DP7.

210 The very high noise level of the MALDI-TOF MS spectrum in negative-ion mode implies 
211 low population of cellulose phosphate. The FTIR spectra shown in Fig. 1 does not detect

212 the existence of phosphorus. EDX measurements yield semi-quantitative assessment of

213 phosphorus by revealing ca. 0.13 at\% of phosphorus in the specimen (Fig. S3). Because of

214 the higher sensitivity of EDX to heavier element phosphorus, and the possibility of

215 electron beam damage to the specimen by selectively vaporizing volatile organic moieties

216 while leaving the phosphorus behind, EDX date do not yield a quantitative measure of the

217 phosphorus composition [35]. The phosphorylation during acid-hydrolysis has not been

218 previously reported, the mechanism and location of phosphate bond remain uncertain,

219 further studies are needed.

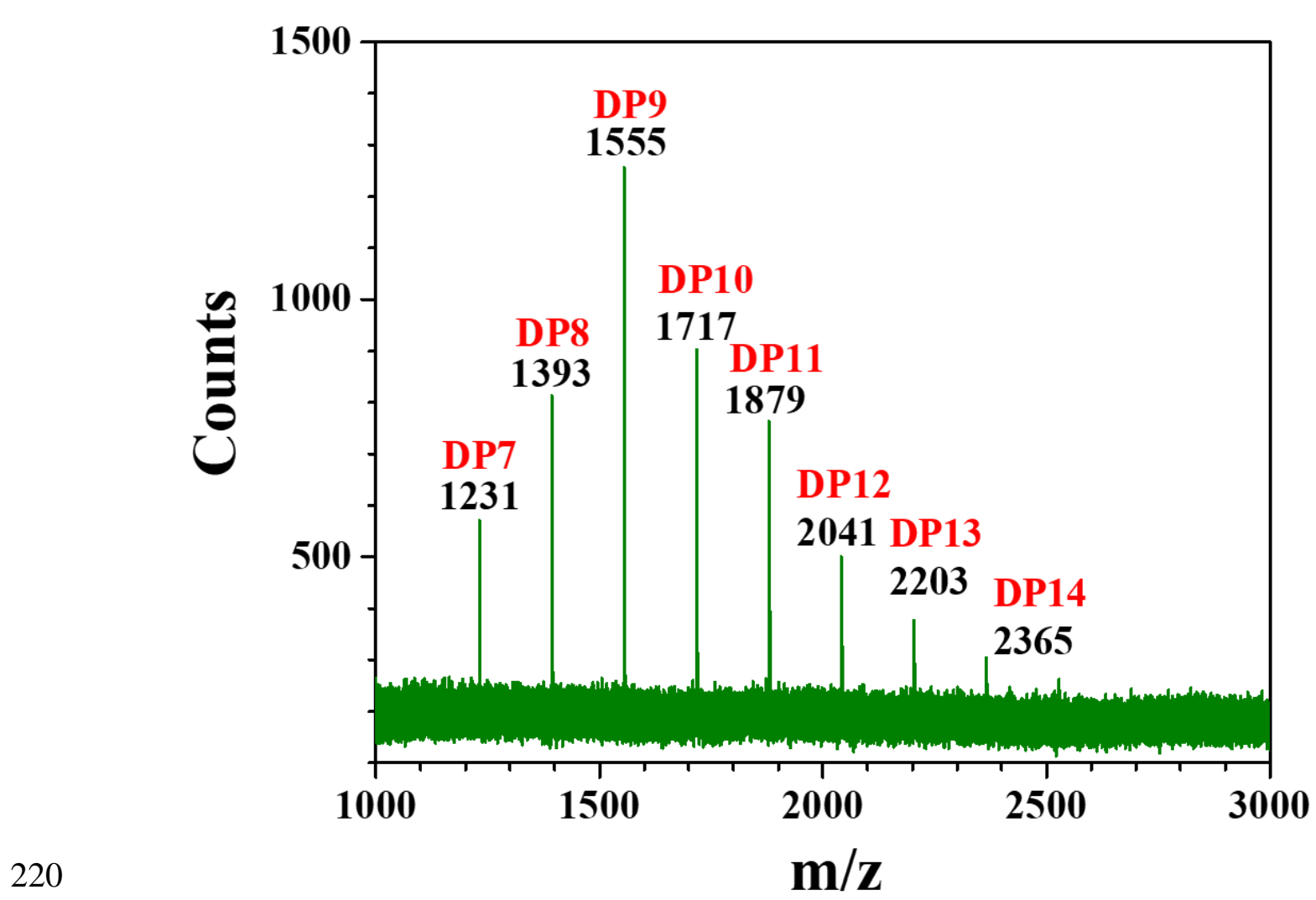


221 Fig. 5. Negative-ion mode MALDI-TOF MS spectrum of the oligocellulose DP7. The

222 molecular mass quantity and corresponding DPs are marked on top of each mainline. The

223 DP has a range of 7-15 and peaks at 9. Note the very high noise level for measuring

224 negative ion species.

225

226 3.3. SAXS and SANS analysis

227 SAXS and SANS patterns of 1, 2 and 5 wt $\%$ of DP7 in 4 wt $\%$ of LiOH aqueous

228 solutions, as well as 1 wt $\%$ DP7 in dDMSO are shown in Fig. 6. The plateau at low- $Q$

229 region implies cellulose oligomer solutions without aggregation in both alkaline and

230 organic solvents. A medium- $Q$ region with a power-law of $Q^{-1}$ indicates $1 D$-like structure,

231 i.e. extended conformation of DP7 in alkaline solution, followed by a transient $Q^{-2}$ scaling

232 in the high- $Q$ region due to the flat shape of glucose residue. The radius of gyration $\left(R_{\mathrm{g}}\right)$ of

233 dispersed particles is analyzed from the low- $Q$ region of small angle scattering using

234 Guinier law, $I(Q)=I_{0} \exp \left(-Q^{2} R_{g}^{2} / 3\right)$, where $I_{0}$ is the zero angle scattering at $Q=0$.

235 Linearized plot with fitting as shown in the inset of Fig. 6 yields $R_{\mathrm{g}}=10.2 \pm 0.3 \AA$ for the 5

236 wt \% DP7. Same analyses have been applied to 1 and 2 wt $\%$ solutions to yield $R_{\mathrm{g}}$ 's at 10.5

$237 \pm 0.4 \AA$ and $11.0 \pm 0.6 \AA$, respectively. Due to deprotonation of the hydroxyl groups to

238 afford charged side chains at high $\mathrm{pH}[36]$ and the intrinsically rigid backbones, cellulose

239 oligomers can be assumed to take rigid rod-like conformation. Using a model of cylindrical

240 rod with a radius $R=3 \AA$, the average length of DP7 is estimated from $R_{\mathrm{g}}$ with equation, 
$241 L^{2}=12 R_{g}^{2}-6 R^{2}$, yielding $L=36 \AA$. Given the length of AGU at $5.15 \AA$ [37-40], the

242 average DP $~ 7$ is consistent with the NMR and MALDI-TOF MS results.

243 The scattering curves over the entire $Q$-range were also fitted with a parallelepiped

244 model using SASView 5.0; the best fitting curves are plotted on the experimental data in

245 Fig. 6, and the best fit parameters are listed in the Table S1. The DP7 chains in LiOH

246 aqueous solutions can be described as a rigid slab of thickness $\sim 4 \AA$, width $\sim 8 \AA$, and

247 lengths about 34-42 A. Using the same slab model with fixed thickness and width

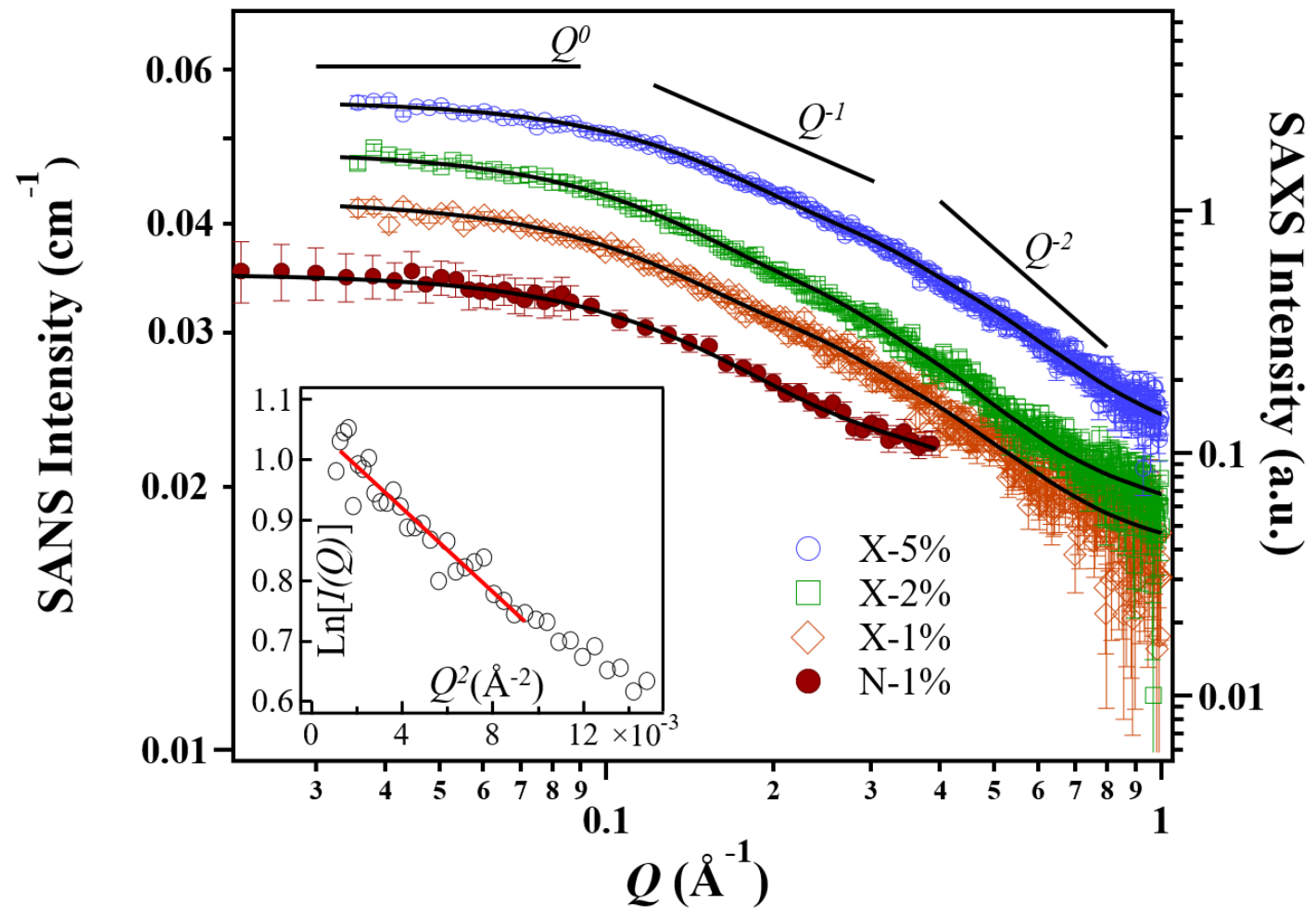

248 quantities from SAXS, fitting to SANS (curve through the data in Fig. 6) yields $L=29 \pm 3$

$249 \AA$ A, slightly smaller than those from SAXS. The fitted length from SAXS/SANS data 250 corresponds to a DP range of 5.6-8.2, consistent with other measurements. 
251 Fig. 6. Experimental data of SAXS on 1 wt $\%$ (X-1\%, open diamonds), 2 wt \% (X-2\%,

252 open squares), and $5 \mathrm{wt} \%$ (X-5\%, open cycles) DP7 in $4 \mathrm{wt} \%$ of LiOH and SANS on $1 \mathrm{wt} \%$

253 DP7 in dDMSO (N-1\%, filled circles) are shown as symbols with error bars from counting

254 statistics, and the solid curves through symbols are best model fitting calculation. The inset

255 shows Guinier analysis of the SAXS on the $5 \mathrm{wt} \%$ DP7 in alkaline solution.

\section{4. Conclusions}

258 We have revisited the classical method of producing oligocellulose through hydrolysis

259 of microcrystalline cellulose in phosphoric acid at room temperature. The oligocellulose

260 products were overall of high purity but also contained a small fraction of

261 mono-substituted cellulose phosphate possibly from the side-reaction of the hydrolysis

262 process. The overall production yield of oligocellulose was about 50\%. The lower DP

263 fraction is composed of cellulose molecules from DP5 to DP12, with DP7 being the most

264 abundant species. The number- and weight-averaged molecular weights as measured from

265 the mass spectra were $1339 \mathrm{~g} / \mathrm{mol}$ and $1388 \mathrm{~g} / \mathrm{mol}$ respectively, corresponding to the

266 polydispersity index of 1.04. They form type-II crystals in solid powder, and dissolve in

267 alkaline aqueous solutions and dimethyl sulfoxide. Although small in quantity,

268 mono-phosophorylated oligomers were also detected. SAXS and SANS measurements of

269 the solution agreed with theoretical curves of slab object indicating the extended

270 conformation of cellulose oligomers. 


\section{Declaration of Competing Interest}

273 The authors declare no conflict of interest.

274

\section{Acknowledgements}

276 F. Jiang was financially supported by the National Natural Science Foundation of China

277 (Grant 51603199), China Postdoctoral Science Foundation (Grant 2017M622629), and

278 Anhui Provincial Innovation and Entrepreneurship Support Plan for Overseas Returnees

279 (Grant 2020LCX027). We acknowledge the support of the National Institute of Standards

280 and Technology through award 70NANB12H238 and by providing the neutron research

281 facilities used in this study. This work benefited from the use of the SasView application,

282 originally developed under an NSF award DMR-0520547. SasView contains code

283 developed with funding from the European Union's Horizon 2020 research and innovation

284 program under the SINE2020 project, grant agreement No. 654000. We thank Dr. Yimin

285 Mao of NCNR/NIST for help on SANS experiment and data reduction.

287 Appendix A. Supplementary material

288 Supplementary material associated with this article is available.

290 References 
291 [1] H. Kargarzadeh, M. Mariano, D. Gopakumar, I. Ahmad, S. Thomas, A. Dufresne, J.

292 Huang, N. Lin, Advances in cellulose nanomaterials, Cellulose 25 (2018) 2151-2189.

293 [2] S. Wang, A. Lu, L.N. Zhang, Recent advances in regenerated cellulose materials, Prog.

294 Polym. Sci. 53 (2016) 169-206.

295 [3] F. Jiang, Z.K. Wang, Y.L. Qiao, Z.G. Wang, C.B. Tang, A novel architecture toward 296 third-generation thermoplastic elastomers by a grafting strategy, Macromolecules 46 297 (2013) 4772-4780.

298 [4] S. Agate, M. Joyce, L. Lucia, L. Pal, Cellulose and nanocellulose-based 299 flexible-hybrid printed electronics and conductive composites-a review, Carbohydr. 300 Polym. 198 (2018) 249-260.

301 [5] O. Garcia-Valdez, P. Champagne, M.F. Cunningham, Graft modification of natural 302 polysaccharides via reversible deactivation radical polymerization, Prog. Polym. Sci. 76 303 (2018) 151-173.

304 [6] J.S. Yang, J.F. Li, Self-assembled cellulose materials for biomedicine: a review, 305 Carbohydr. Polym. 181 (2018) 264-274.

306 [7] F. Jiang, C.Q. Pan, Y.Q. Zhang, Y.X. Fang, Cellulose graft copolymers toward strong 307 thermoplastic elastomers via RAFT polymerization, Appl. Surf. Sci. 480 (2019) 162-171.

308 [8] M. Meiland, T. Liebert, T. Heinze, Tailoring the degree of polymerization of low 309 molecular weight cellulose, Macromol. Mater. Eng. 296 (2011) 802-809.

310 [9] S.I. Mussatto, I.M. Mancilha, Non-digestible oligosaccharides: a review, Carbohydr. 
311 Polym. 68 (2007) 587-597.

312 [10] P.R. Chen, A. Shrotri, A. Fukuoka, Soluble cello-oligosaccharides produced by

313 carbon-catalyzed hydrolysis of cellulose, ChemSusChem 12 (2019) 2576-2580.

314 [11] F. Nakatsubo, H. Kamitakahara, M. Hori, Cationic ring-opening polymerization of 3,

315 6-di- $O$-benzyl- $\alpha$ - $D$-glucose 1,2,4-orthopivalate and the first chemical synthesis of

316 cellulose, J. Am. Chem. Soc. 118 (1996) 1677-1681.

317 [12] S. Kobayashi, K. Kashiwa, T. Kawasaki, S. Shoda, Novel method for polysaccharide

318 synthesis using an enzyme: the first in vitro synthesis of cellulose via a nonbiosynthetic

319 path utilizing cellulase as catalyst, J. Am. Chem. Soc. 113 (1991) 3079-3084.

320 [13] C.J. Knill, J.F. Kennedy, Degradation of cellulose under alkaline conditions,

321 Carbohydr. Polym. 51 (2003) 281-300.

322 [14] R. Gupta, Y. Lee, Mechanism of cellulase reaction on pure cellulosic substrates,

323 Biotechnol. Bioeng. 102 (2009) 1570-1581.

324 [15] T. Zweckmair, J.T. Oberlerchner, S. Bohmdorfer, M. Bacher, V. Sauerland, T.

325 Rosenau, A. Potthast, Preparation and analytical characterisation of pure fractions of

326 cellooligosaccharides, J. Chromatogr. A 1431 (2016) 47-54.

327 [16] E. Billes, K.N. Onwukamike, V. Coma, S. Grelier, F. Peruch, Cellulose oligomers

328 production and separation for the synthesis of new fully bio-based amphiphilic

329 compounds, Carbohydr. Polym. 154 (2016) 121-128.

330 [17] M. Voloch, M.R. Ladisch, M. Cantarella, G.T. Tsao, Preparation of cellodextrins 
331 using sulfuric acid, Biotechnol. Bioeng. 26 (1984) 557-559.

332 [18] O. Akpinar, R.J. McGorrin, M.H. Penner, Cellulose-based chromatography for

333 cellooligosaccharide production, J. Agric. Food Chem. 52 (2004) 4144-4148.

334 [19] T. Liebert, M. Seifert, T. Heinze, Efficient method for the preparation of pure,

335 water-soluble cellodextrines, Macromol. Symp. 262 (2008)140-149.

336 [20] A. Isogai, M. Usuda, Preparation of low-molecular weight celluloses using

337 phosphoric acid, Mokuzai Gakkaishi 37 (1991) 339-344.

338 [21] M. Schwanninger, J.C. Rodrigues, H. Pereira, B. Hinterstoisser, Effects of short-time

339 vibratory ball milling on the shape of FT-IR spectra of wood and cellulose, Vib. Spectrosc.

$34036(2004) 23-40$.

341 [22] Y. Yataka, T. Sawada, T. Serizawa, Enzymatic synthesis and post-functionalization of

342 two-dimensional crystalline cellulose oligomers with surface-reactive groups, Chem.

343 Commun. 51 (2015) 12525-12528.

344 [23] Z.H. Liu, X.F. Sun, M.Y. Hao, C.Y. Huang, Z.M. Xue, T.C. Mu, Preparation and

345 characterization of regenerated cellulose from ionic liquid using different methods,

346 Carbohydr. Polym. 117 (2015) 99-105.

347 [24] C.-H. Kuo, C.-K. Lee, Enhancement of enzymatic saccharification of cellulose by

348 cellulose dissolution pretreatments, Carbohydr. Polym. 77 (2009) 41-46.

349 [25] M. Adsul, S.K. Soni, S.K. Bhargava, V. Bansal, Facile approach for the dispersion of 350 regenerated cellulose in aqueous system in the form of nanoparticles, Biomacromolecules 
352 [26] S.Y. Oh, D.I. Yoo, Y. Shin, H.C. Kim, H.Y. Kim, Y.S. Chung, W.H. Park, J.H. Youk,

353 Crystalline structure analysis of cellulose treated with sodium hydroxide and carbon

354 dioxide by means of X-ray diffraction and FTIR spectroscopy, Carbohydr. Res. 340 (2005)

$355 \quad 2376-2391$.

356 [27] P. Langan, Y. Nishiyama, H. Chanzy, A revised structure and hydrogen-bonding

357 system in cellulose II from a neutron fiber diffraction analysis, J. Am. Chem. Soc. 121

358 (1999) 9940-9946.

359 [28] P. Langan, Y. Nishiyama, H. Chanzy, X-ray structure of mercerized cellulose II at 1

360 A resolution, Biomacromolecules 2 (2001) 410-416.

361 [29] L.A. Flugge, J.T. Blank, P.A.J.J.o.t.A.C.S. Petillo, Isolation, modification, and NMR

362 assignments of a series of cellulose oligomers, J. Am. Chem. Soc. 121 (1999) 7228-7238.

363 [30] B. Bernet, A. Vasella, Intra- and intermolecular H-bonds of alcohols in DMSO,

$364{ }^{1} \mathrm{H}-\mathrm{NMR}$ analysis of inter-residue H-bonds in selected oligosaccharides: cellobiose,

365 lactose, $N, N^{\prime}$-diacetylchitobiose, maltose, sucrose, agarose, and hyaluronates, Helv. Chim.

366 Acta 83 (2000) 2055-2071.

367 [31] H. Sugiyama, K. Hisamichi, T. Usui, K. Sakai, J. Ishiyama, A study of the

368 conformation of $\beta$-1,4-linked glucose oligomers, cellobiose to cellohexaose, in solution, J.

369 Mol. Struct. 556 (2000) 173-177.

370 [32] J.S. Moulthrop, R.P. Swatloski, G. Moyna, R.D.J.C.c. Rogers, High-resolution ${ }^{13}$ C 
371 NMR studies of cellulose and cellulose oligomers in ionic liquid solutions, Chem.

372 Commun. (2005) 1557-1559.

373 [33] D.M. Petrovic, I. Kok, A.J.J. Woortman, J. Ciric, K. Loos, Characterization of

374 oligocellulose synthesized by reverse phosphorolysis using different cellodextrin

375 phosphorylases, Anal. Chem. 87 (2015) 9639-9646.

376 [34] A. Adharis, D.M. Petrović, I. Özdamar, A.J. Woortman, K. Loos, Environmentally

377 friendly pathways towards the synthesis of vinyl-based oligocelluloses, Carbohydr.

378 Polym. 193 (2018) 196-204.

379 [35] A.R. Horrocks, Textile flammability research since 1980-personal challenges and 380 partial solutions, Polym. Degrad. Stab. 98 (2013) 2813-2824.

381 [36] A. Isogai, R.H. Atalla, Dissolution of cellulose in aqueous $\mathrm{NaOH}$ solutions, 382 Cellulose 5 (1998) 309-319.

383 [37] J. Cai, Y.T. Liu, L.N. Zhang, Dilute solution properties of cellulose in LiOH/urea 384 aqueous system, J. Polym. Sci., Part B: Polym. Phys. 44 (2006) 3093-3101.

385 [38] J. Hagman, L. Gentile, C.M. Jessen, M. Behrens, K.E. Bergqvist, U. Olsson, On the 386 dissolution state of cellulose in cold alkali solutions, Cellulose 24 (2017) 2003-2015.

387 [39] X.Y. Jiang, S. Kitamura, T. Sato, K. Terao, Chain dimensions and stiffness of 388 cellulosic and amylosic chains in an ionic liquid: cellulose, amylose, and an amylose 389 carbamate in BmimCl, Macromolecules 50 (2017) 3980-3985.

390 [40] J.P. Zhou, L.N. Zhang, J. Cai, Behavior of cellulose in NaOH/urea aqueous solution 
391 characterized by light scattering and viscometry, J. Polym. Sci., Part B: Polym. Phys. 42

392 (2004) 347-353.

393 